\title{
The L-PRF Membrane (Fibrin Rich in Platelets and Leukocytes) and Its Derivatives (A-PRF, I-PRF) Are Useful as a Source of Stem Cells in Regenerative Wound Therapy: Experimental Work on the Horse
}

\author{
Alessandro Crisci $i^{1,2,3^{*}}$ \\ ${ }^{1}$ School of Medicine, University of Salerno, Italy \\ 2Unit of Dermosurgery Cutaneous Transplantations and Hard-to-Heal Wound, "Villa Fiorita"Private Hospital, Italy \\ ${ }^{3}$ Institute for the Studies and Care of Diabetics, Italy
}

\begin{abstract}
Growing multidisciplinary field of tissue engineering aims to regenerate, improve or replace predictably damaged or missing tissues for a variety of conditions caused by trauma, disease and old age. To ensure that tissue engineering methods are widely applicable in the clinical setting, it is necessary to modify them in such a way that they are readily available and relatively easy to use in daily clinical routine. Therefore, the steps between preparation and application must be minimized and optimized to make them realistic implementation. General objective of developing platelet concentrates of natural origin, can be produced close to the patient and accelerate the implantation process, being financially realistic for the patient and the health system. Fibrin rich in platelets and leukocytes (PRF) and its derivatives ( $L-P R F, A-P R F, i-P R F)$ have been used in a wide variety of medical fields for soft tissue regeneration. Almost all platelets $(>97 \%)$ are absent inside test-tubes in groups tested after PRF membrane extraction.
\end{abstract}

Growth Factors freed by platelets contained in derivatives of L-PRF induce and control the proliferation and migration of other cell types, involved in tissue repair, like smooth cell muscles (SMCs) and mesenchymal stem cells (MSCs).

In conclusion, the results of this work highlight the positive effects of PRF on wound healing after regenerative therapy for the management of various soft tissue defects found in wound care.

\section{Keywords}

Blood derivatives, Growth factors, Leucocyte and platelets-rich fibrin, Injectable platelets-rich fibrin, L-PRF wound box, Stem cells

\section{Introduction}

Recent scientific evidence suggests that platelets could play a new role in tissue repair and in vascular remodeling, other than being active actors in inflammatory and immune responses. Platelets release biologically active proteins and other substances, able to influence a series of processes, favoring cell intake, growth and morphogenesis. These substances are released or exposed on the surface of activated platelets. The platelet capability of releasing substances inside a clot makes the clot itself a natural autologous source of growth factors and cytokines, which can be therapeutically used to accelerate and speed up physiological healing processes. Many of these substances are gathered and stored in platelets $\alpha$-granules, easily identified with scanning electron microscope (SEM) and with immunofluorescence staining.

The fine fibrin fibers contained in HPC (Human Platelet
Concentrate) could be related to the high initial concentration of platelets in HPC $\left(3-5 \times 10^{11}\right.$ platelets/l), where local procoagulant activity could even be improved through the start of pro-thrombotic stimuli amplification, and it leads to an almost explosive thrombin production, thus causing an increase in fibrinogenesis on the surface of platelets, which in

*Corresponding author: Prof. Alessandro Crisci, School of Medicine, University of Salerno, Fisciano (SA), Italy

Accepted: August 29, 2019

Published online: August 31, 2019

Citation: Crisci A (2019) The L-PRF Membrane (Fibrin Rich in Platelets and Leukocytes) and Its Derivatives (A-PRF, I-PRF) Are Useful as a Source of Stem Cells in Regenerative Wound Therapy: Experimental Work on the Horse. Regen Med Ther $3(1): 37-45$ 
Citation: Crisci A (2019) The L-PRF Membrane (Fibrin Rich in Platelets and Leukocytes) and Its Derivatives (A-PRF, I-PRF) Are Useful as a Source of Stem Cells in Regenerative Wound Therapy: Experimental Work on the Horse. Regen Med Ther 3(1):37-45

turn leads to fibrin formation and polymerization [1].

The introduction of blood concentrates such as platelet-rich plasma and fibrin (PRP/PRF) was the first step toward meeting clinical requirements [2] (Figure 1). This blood concentrate is derived from the patient's peripheral blood after a one-step centrifugation without anticoagulants to generate a platelet- and leucocyte-rich matrix. The presence of platelets, leucocytes and fibrin was previously shown to be essential for wound healing $[3,4]$. In addition to the potential of leucocytes to influence angiogenesis and lymphomagenesis, this fibrin network, containing leucocytes and platelets, is a source of cytokines and growth factors, which are main players in the process of wound healing [5]. The use of specific plastic tubes favors non-clotting PRF and resulted in the development of a liquid PRF-based matrix (liquid-PRF) produced without the need for anticoagulants. Previously, a systematic study investigated the influence of the applied relative centrifugal force (RCF) on the composition and bioactivity of PRF-matrices.

This fibrin scaffold, which has no cytotoxic potential, is obtained from $9 \mathrm{ml}$ of the patient's blood after 1 phase of centrifugation and contains a variety of blood cells-including platelets, B and T lymphocytes, monocytes, stem cells, and neutrophil granulocytes-in addition to growth factors. Furthermore, L-PRF (also called leukocyte-PRF) contains white blood cells, necessary cells that are important during the wound healing process [6]. Moreover, since white blood cells, including neutrophils and macrophages, are among the first types of cells present in wound sites, their role also includes

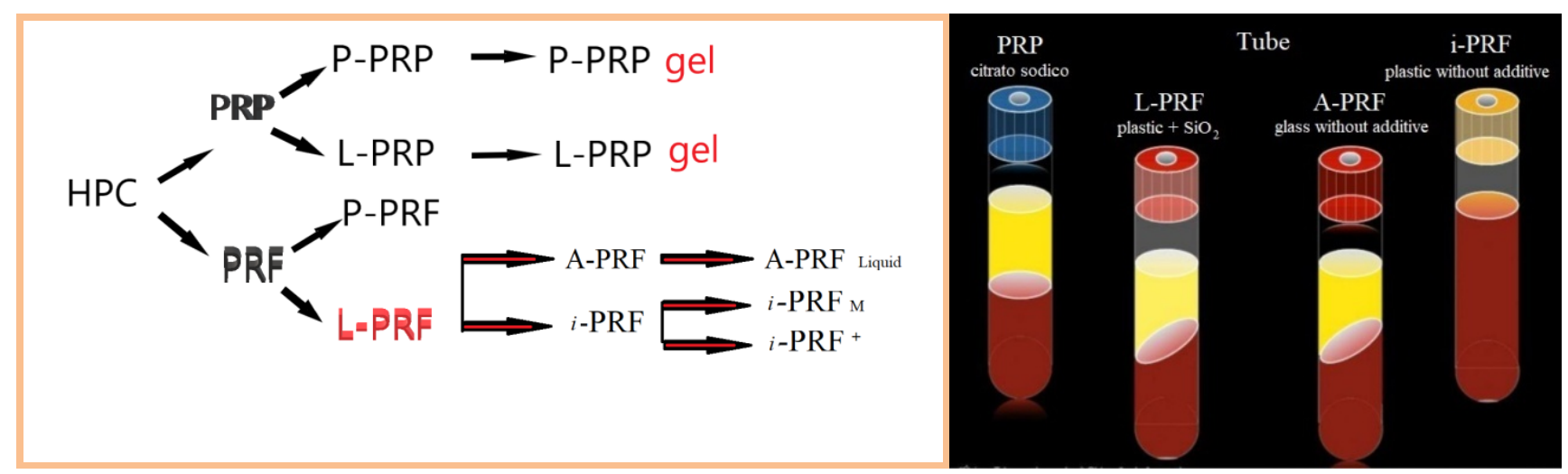

Figure 1: Different types of human platelet concentrates (HPCS): PRP (Platelet-rich plasma); PRF (Platelet-rich fibrin); P-PRP (Pure Platelet-rich plasma); L-PRP (Leukocyte and Platelet-rich plasma); P-PRF (Pure Platelet-rich fibrin); L-PRF (Leukocyte and Platelet-rich fibrin); $i$-PRF (Injectable Platelet-rich fibrin); A-PRF (Advanced Platelet-rich fibrin).

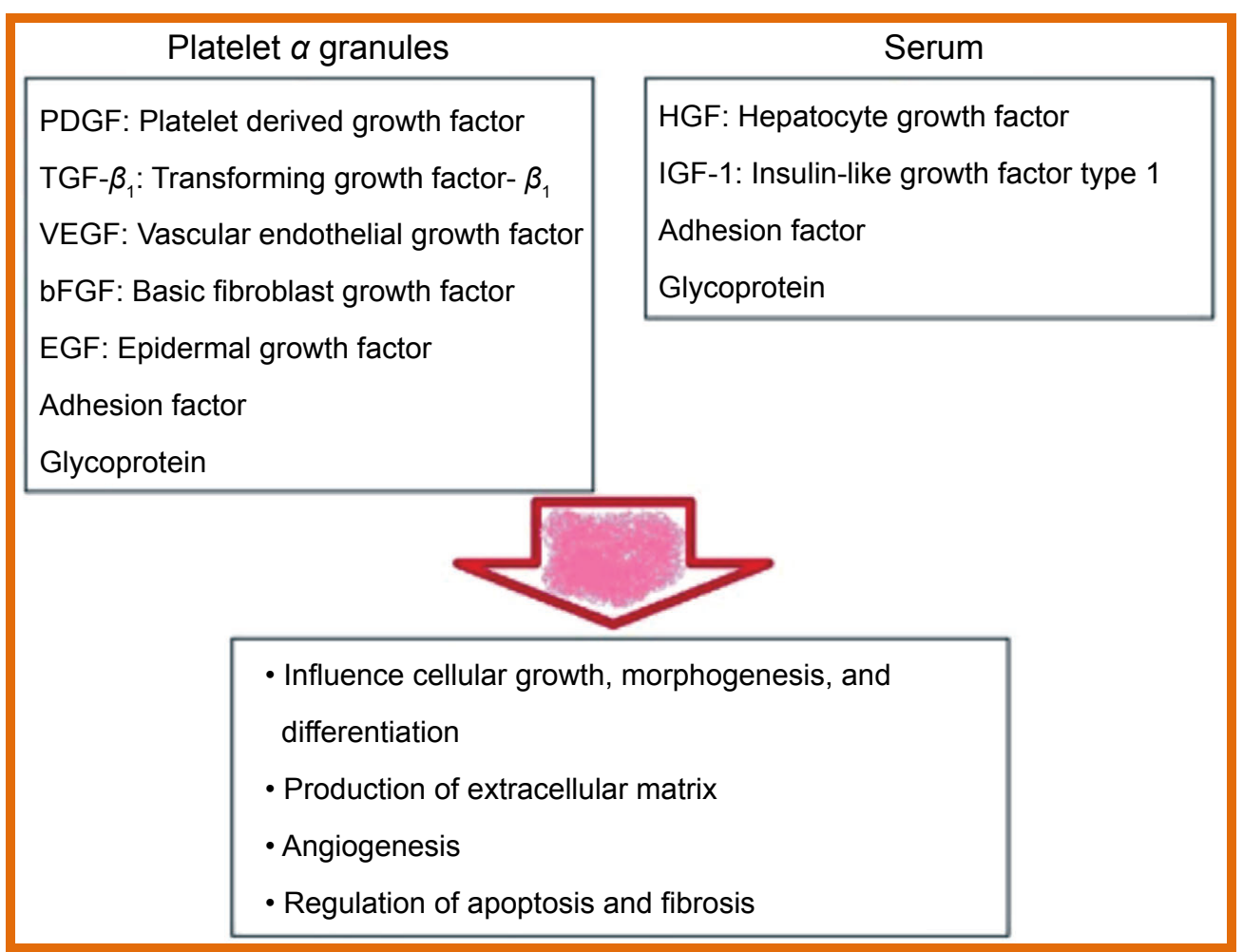

Figure 2: Function of the platelets in wound healing. 
Citation: Crisci A (2019) The L-PRF Membrane (Fibrin Rich in Platelets and Leukocytes) and Its Derivatives (A-PRF, I-PRF) Are Useful as a Source of Stem Cells in Regenerative Wound Therapy: Experimental Work on the Horse. Regen Med Ther 3(1):37-45

phagocytic fragments, microbes, and necrotic tissue, thus preventing infection. Macrophages are also key cells derived from the myeloid lineage and are considered one of the key cells involved in growth factor secretion during wound healing, including the transforming growth factor beta (TGF- $\beta$ ), PDGF and growth factor vascular endothelium (VEGF) (Figure 2). These cells, together with neutrophils and platelets, are key players in wound healing and in combination with their growth factors/secreted cytokines are able to facilitate tissue regeneration, the formation of new blood vessels (angiogenesis) and the infection prevention.

\section{L-PRF}

In the longitudinal section of the L-PRF coagulum, produced according to the standard centrifugation protocol (30" of acceleration, 2' at $2700 \mathrm{rpm}, 4^{\prime}$ at $2400 \mathrm{rpm}, 3^{\prime}$ at $3000 \mathrm{rpm}$, and 36 " of deceleration and stopping) [6], a thick fibrin clot is present with minimal inter-fibre space. Cells are observed throughout the blood clot, although decreasing towards the most distal parts of the PRF clot (Figure 1 and Figure 3).

\section{Advanced-PRF}

The PRF clots formed with the A-PRF centrifugation protocol (Advanced-PRF) (1500 rpm, 14 minutes) [7] showed a freer structure with more inter-fibre space and more cells can be counted in the fibrin-rich clot. Furthermore, the cells are more evenly distributed in the clot than L-PRF, and some cells can also be found in the most distal parts of the clot. A representative image for cellular distribution within A-PRF is shown in Figure 4.

\section{PRF injectable formulation}

The development of an injectable formulation of PRF (referred to as $i$-PRF) $[8,9]$ (centrifuged at $700 \mathrm{rpm}(60 \mathrm{~g})$ for 3 minutes) was pursued with the goal of delivering a platelet concentrate easy to use to doctors in liquid formulation that can be used alone or easily combined with various biomaterials. Taking advantage of slower and shorter centrifugation speeds, a greater presence of regenerative cells with higher concentrations of growth factors can be observed compared to other PRF formulations using higher centrifugation rates. Ghanaati, et al. [7] reported that velocity and time do not affect monocyte and stem cell concentrations, but influence platelet and neutrophil concentrations. As a result, A-PRF

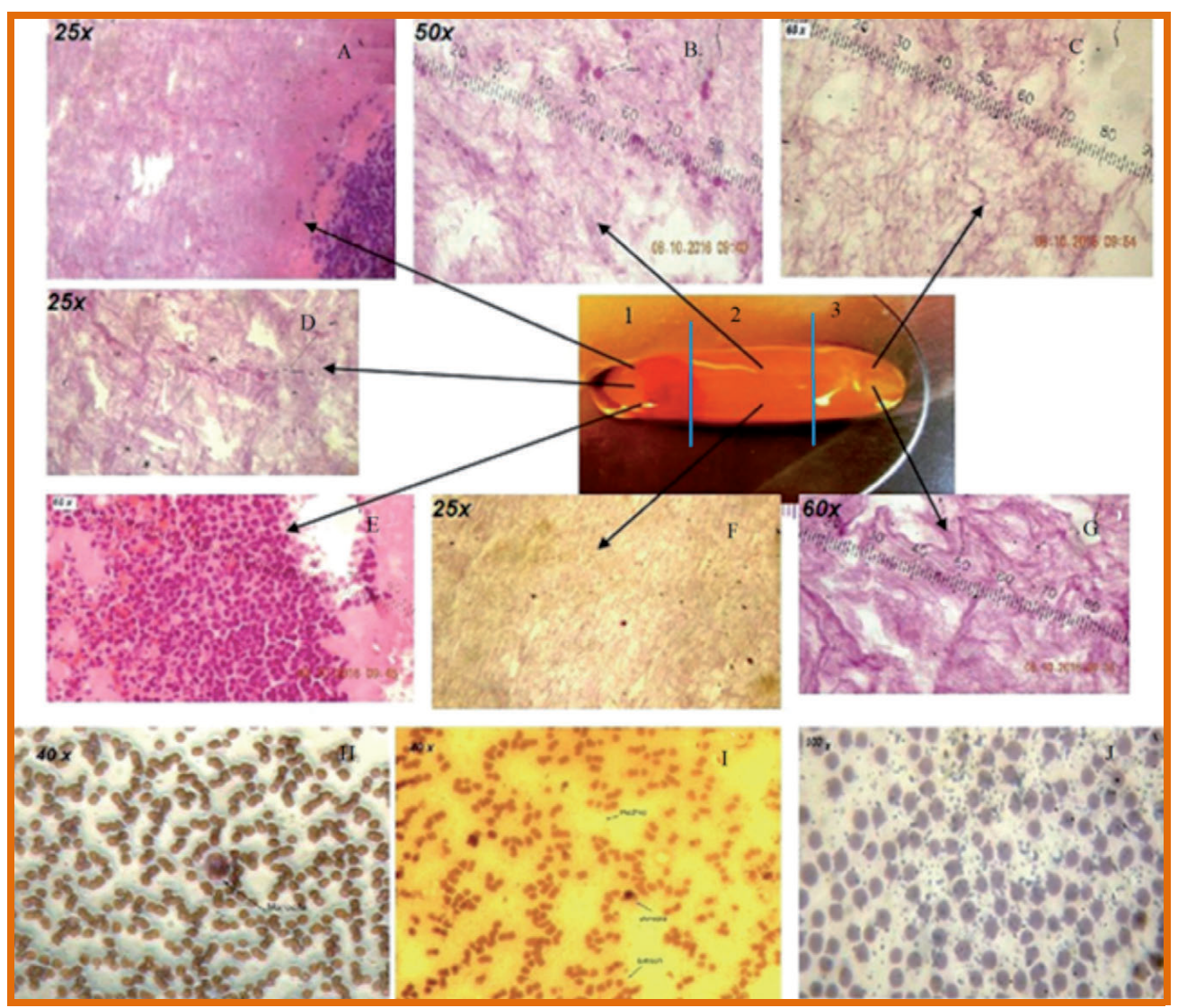

Figure 3: Horse L-PRF membrane at 0 minutes from compression (eosin-hematoxylin color). The L-PRF layers were fixed in $10 \%$ formalin buffered neutral solution at $\mathrm{pH} 7.2$ for 48 hours and incorporated in paraffin according to the standard procedure. Twenty serial sections ( $7 \mu \mathrm{m}$ thickness) of each sample were cut using a microtome. A) III proximal ingr. $25 \times$ white blood cells-fibrin reticulum; B) III average ingr. $60 \times$ erythrocytes-fibrin pattern; C) III distal ingr. $60 \times$ fibrin reticulum; D) III proximal ingr. $25 \times$ erythrocytes-fibrin; E) III proximal ingr. $60 \times$ fibrin on the right, lymphocytes in the center, erythrocytes and neutrophil granulocytes on the left; F) III medium ingr. $25 \times$ fibrin lattice; G) III distal ingr. $60 \times$ fibrin reticulum; H) Red clot smear ingr. $40 \times$ presence of monocita in a carpet of erythrocytes; I) Smear red clot ingr. $40 \times$ presence of erythrocytes, monocytes and platelets; J) Smear red clot ingr. $100 \times$ platelets in a carpet of erythrocytes (May-Grǔnwald-Giemsa stain). Reproduced from Crisci, et al. [4] licensed under the terms of Creative Commons Attribution 4.0 International License. 


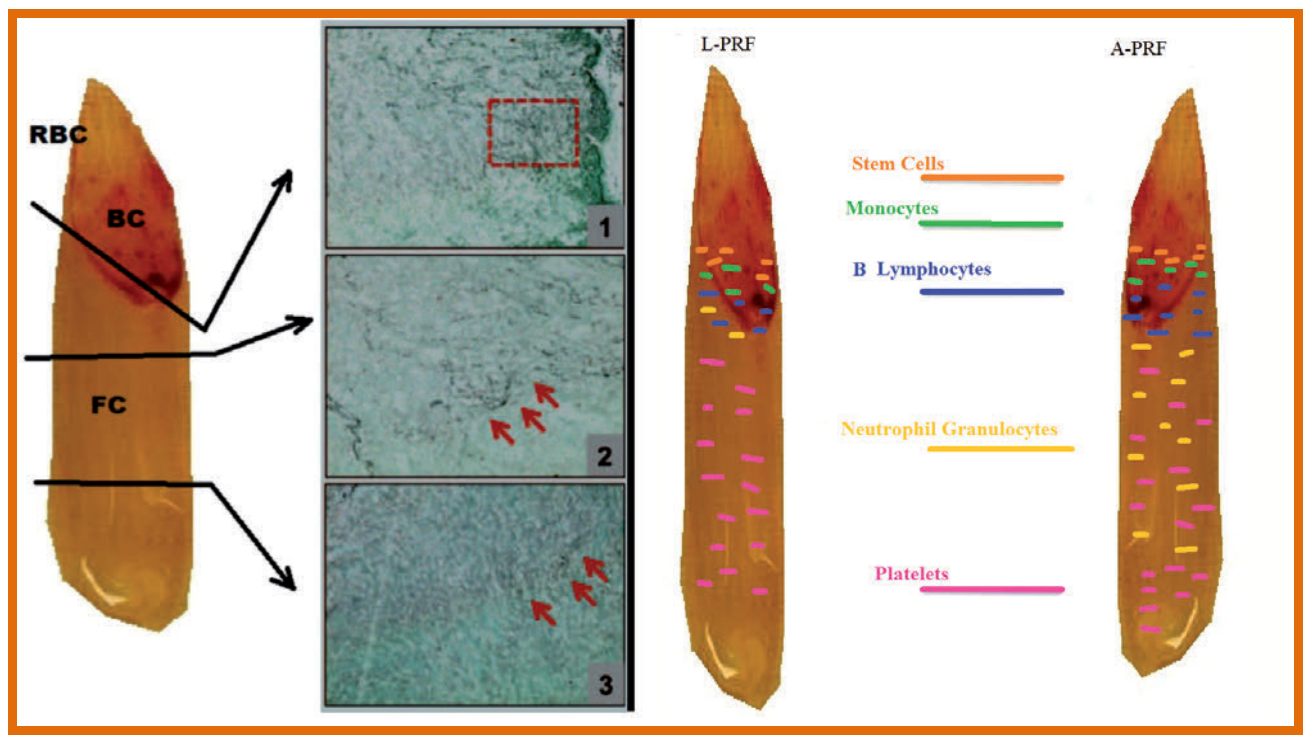

Figure 4: Advanced-PRF (A-PRF) total scan of a fibrin clot along its longitudinal axis (Masson-Goldner staining). RBC represents the fraction of red blood cells. The buffy coat $(\mathrm{BC})$ is the transformation zone between the fraction of RBC and the fibrin clot and FC represents the fibrin clot. The three bars within the scan and the arrows show the first floors of the respective areas. The red arrows mark cells that are trapped inside the fibrin network.

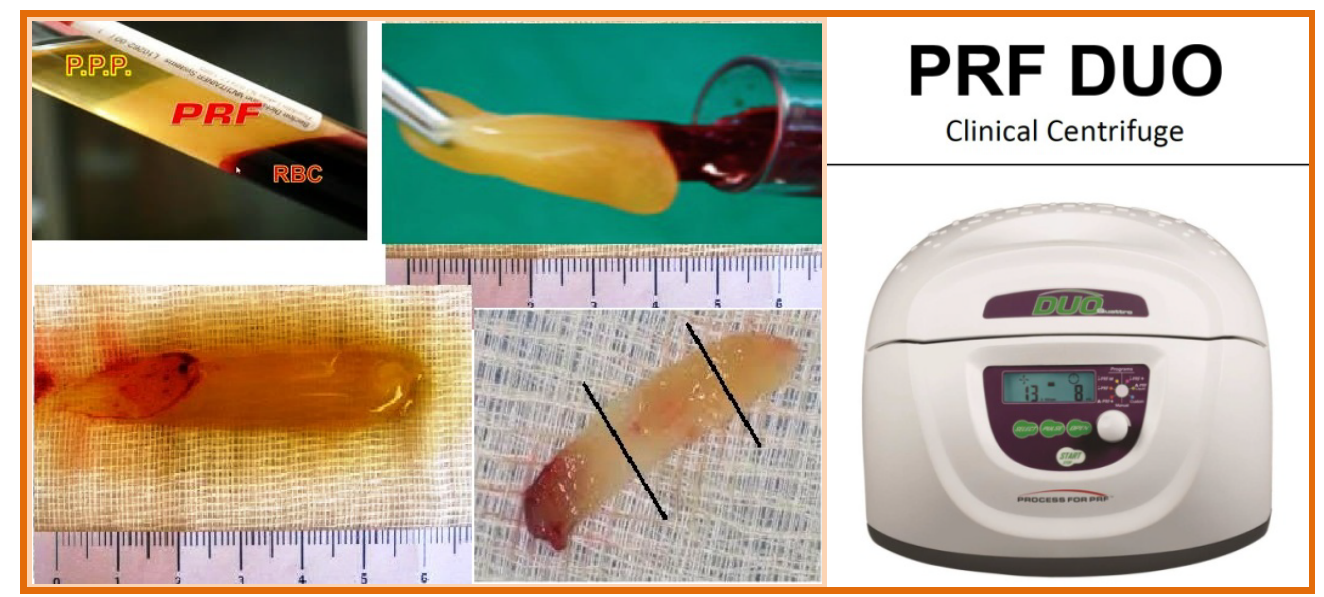

Figure 5: Various stages of preparation of the L-PRF (see text) with Duo centrifuge (Process for PRF, France).

contains more platelets, most were found in the distal part of the PRF and membrane include more neutrophils. This type of concentrate has the potential to improve angiogenesis by expressing the enzymatic matrix metalloproteinase- 9 . Therefore, the inclusion of neutrophils in the PRF could be considered if angiogenesis is of interest. Analysis of the study by Ghanaati, et al. [7] also revealed that the platelets were the only ones present in each coagulum area up to $87 \pm 13 \%$ in the L-PRF group and up to $84 \pm 16 \%$ in the A-PRF group (Figure 4). Furthermore, the results showed that $T$ lymphocytes (L-PRF: $12 \pm 5 \%$, APRF: $17 \pm 9 \%$ ), B lymphocytes (L-PRF: $14 \pm$ $7 \%$, A-PRF: $12 \pm 9 \%$ ), CD34 positive stem cells (L-PRF: $17 \pm 6 \%$, APRF: $21 \pm 11 \%$ ), and Monocytes (L-PRF: $19 \pm 9 \%$, A-PRF: 22 $\pm 8 \%$ ) not more than $30 \%$ of the total length of the clot have been found beyond a certain point, since they are distributed near the $\mathrm{BC}$ generated by the centrifugation process.

\section{Materials and Methods}

\section{L-PRF processing}

L-PRF ${ }^{\circledR}$ production protocol is very simple: Blood is immediately centrifuged within 2 minutes from withdrawal, following the subsequent steps: $30^{\prime \prime}$ of acceleration, $2^{\prime}$ at $2700 \mathrm{rpm}$, $4^{\prime}$ at $2400 \mathrm{rpm}, 3^{\prime}$ at $3000 \mathrm{rpm}$, and 36" of deceleration and arrest. The result product is made of three layers: PPP (Platelet-poor plasma at the top), PRF (central clot), Red blood cells (RBCs) at the bottom, with Duo centrifuge (Process for PRF, France) (Figure 1 and Figure 5). Resulting PRF clots are gathered and red blood cells are removed with the aid of scissors, without macroscopical damage at PRF structure expense.

We performed a prospective study of equine blood, which has been collected in test tubes without anticoagulant plastic-coated glass, nor a gel separator (Vacutainer tubes for se- 
Citation: Crisci A (2019) The L-PRF Membrane (Fibrin Rich in Platelets and Leukocytes) and Its Derivatives (A-PRF, I-PRF) Are Useful as a Source of Stem Cells in Regenerative Wound Therapy: Experimental Work on the Horse. Regen Med Ther 3(1):37-45

rum $9.0 \mathrm{ml}$ ), for the production of $L$-PRF clots and membranes by six healthy horses of various ages (average \pm SD, $10 \pm 4.1$ years, ranging from 4 to $17 \mathrm{yy}$ ), gender, and breed.

A written consent of the owners has been provided for all horses and the blood collection procedure has been performed in accordance with the current AVMA guidelines.

The blood was collected quickly with $9 \mathrm{cc}$ with needle 19G to Vacutainer tubes (22" average value, of less than 25 " per tube) and immediately (within $1 \mathrm{~min}$ ) centrifuged according to the preceding description to a temperature greater than $21^{\circ} \mathrm{C}$. Glass tubes coated with plastic without anticoagulant or gel separator (A-PRF + Vacutainer tubes for Serum $9.0 \mathrm{ml}$ ) were used to produce also A-PRF clots and membranes at an ambient temperature above $21^{\circ} \mathrm{C}\left(21-30{ }^{\circ} \mathrm{C}\right)$.

Fibrinogen is initially concentrate in the middle and superior portion of the test tube, that is in between red blood cells (RBCs) at the bottom and the platelet-poor plasma (PPP) at the top. Clot compression by means of a compression system ( $L-P R F$ box) significantly stimulates cell proliferation and neovascularization [10].

In addition to the standard formulations, PRFs can also be obtained in injectable form ( $i$-PRF). $i$-PRF is obtained by producing a PRF, which is not subsequently compressed. Advantageously, this injectable material can coagulate immediately after injection (within 10-12 minutes) to form a biomaterial and can be combined with any biomaterial of choice for non-covalent incorporation [11].

The PRF was subdivided in 3 regions, of equal length (Figure 3 and Figure 5) and platelet presence in each region was observed through S.E.M., through Optic Microscope in horse-derived preparations, Crisci A, et al. [12].

Region 1 is the region closest to the red clot, and shows a conspicuous number of platelets aggregates, displaying some lymphocytes and other white blood cells. Platelet count is reduced as the distance from the red clot is increased. Inside region 2 (central region), we observe fibrin fibers (primary and secondary fibers) and some platelets. Inside region 3, the fibrin mesh is extremely evident, while the platelet count is low (Figure 3).

Under a clinical point of view, L-PRF and derivatives (A-PRF, i-PRF) shows excellent handling properties: Single
L-PRF clots can be turned into membranes of fitting thickness and dimensions, thanks to the "L-PRF Wound Box" (Figure 6); merging two or more membranes is useful (can also be sutured) to create a bioactive membrane of bigger dimensions, to cover and form bigger grafts. L-PRF membrane can also be cut and tailored. Being flexible enough, it adapts to different anatomical areas.

L-PRF/i-PRF family adapts itself to requirements of the various surgical intervention. Just like clots and membranes, L-PRF has shape and volume easily combined with the great bulk of surgical techniques, as filling method and healing biomaterial apposition, or as protecting membranes for wound healing. Furthermore, it is easy to prepare, also in great quantities, and very cheap, making it particularly apt for everyday clinical practice. It was successfully used by AA, especially in the treatment of cutaneous diabetic ulcers, also with osteomyelitis presence [13].

\section{Macroscopic analysis}

After centrifugation, the L-PRF and A-PRF clots (Figure 5) ware removed from the test tube using sterile tweezers and a smooth spatula to gently release the red clot from the buffy coat. Each L-PRF/A-PRF clot obtained was placed in a tray for measuring the weight and size with a digital scale from a goldsmith and a digital calibre.

The compression of the clot was carried out with the L-PRF Wound Box we designed with a pressure of $142.437 \mathrm{~Pa} / \mathrm{cm}^{2}$ constant for two, five, ten, fifteen minutes (no longer lasting compressions were tested because they did not prove useful in the previous study compared to the two-minute study). This method allowed us to obtain, from each clot, the L-PRF membranes, which were individually weighed and measured with a digital gauge. Finally, they were photographed with a digital camera (Nikon) to be able to calculate the surface in $\mathrm{cm}^{2}$ according to the method we reported in the literature [14]. The surface area in $\mathrm{cm}^{2}$ of clots and the membranes were measured with the Calcderm measurement software [14].

\section{Optical microscopy procedure}

The membranes were fixed in $10 \%$ neutral buffered formalin for $24 \mathrm{~h}$ at ambient temperature for inclusion in paraffin. Subsequent sections of $4 \mu \mathrm{m}$ were performed along the

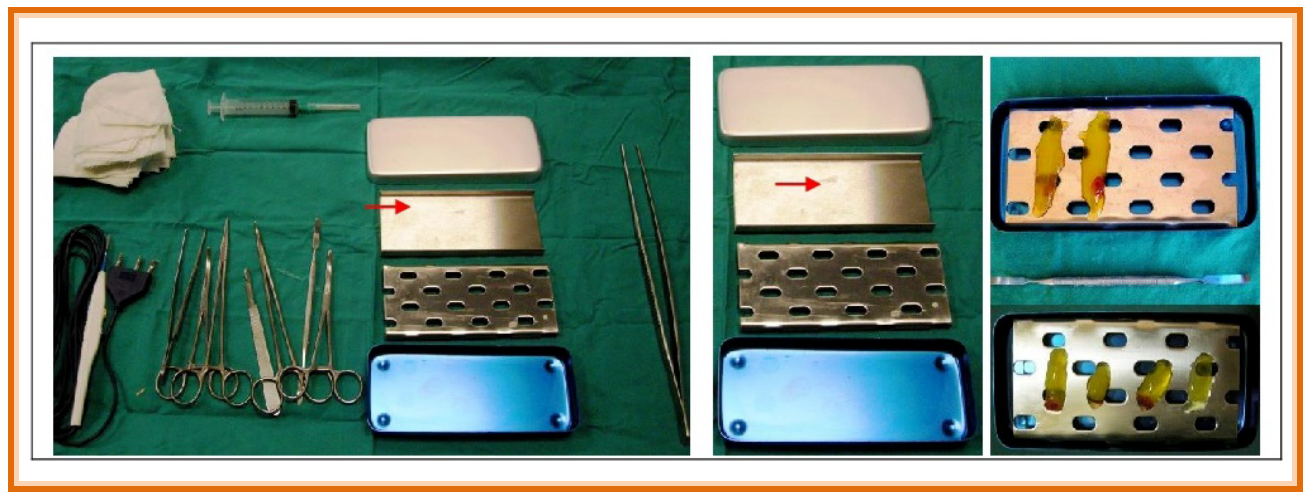

Figure 6: L-PRF wound box. 
Citation: Crisci A (2019) The L-PRF Membrane (Fibrin Rich in Platelets and Leukocytes) and Its Derivatives (A-PRF, I-PRF) Are Useful as a Source of Stem Cells in Regenerative Wound Therapy: Experimental Work on the Horse. Regen Med Ther 3(1):37-45

midpoint of the membranes and were stained with Haematoxylin-Eosin. Each section was divided into three equal-sized areas: Proximal (head), centre (body), and distal (tail). Each area of these sections was observed through an optical microscope (Kern OBN-148) and analyzed by counting the visible cell bodies (marked in dark purple, mostly leukocytes) at the centre of each observed area with a magnification of $25 \times, 40$ $x, 60 \times$, and $100 \times$ (immersion). The total number of counted cell bodies were used to correlate their distribution between the three membrane areas (head, body, and tail). Most of the cells were concentrated in the proximal area (head), closest to the red clot.

Smears of blood prepared by residual blood in the tubes were also examined for a morphological assessment after removal of the PRF clot with a spatula (two for each tube), differentiating the clot at 0 min by centrifugation and fixing them with alcohol $90 \%$ for a Grǔnwald-May-Giemsa colouring stain in order to identify the various corpuscular elements, in particular platelets and neutrophils, to compare them in relation to the examination blood count.

A blood samples was also taken from each horse to perform a blood count using K3E 5.4 mg EDTA tubes (VacuMed).

The supernatant derived from compression with the Wound Box membranes L-PRF and A-PRF was differentiated between the 0 min and was been preserved in a test tube with K3E 5.4 mg EDTA for blood count analysis. It was compared with the basal one and with the corpuscular elements of the counts performed on smears derived from the red clot as an indirect measurement of the platelet and the leukocyte concentrations of the L-PRF.

The supernatant derived from the pressing of L-PRF/A-PRF at 0 min were analysed with a blood count in standard lodging. Since a direct measurement of platelet concentration of the PRF is not yet possible, we calculated the residual platelet concentration with the subtraction method according to the following formula from Watanabe, et al. [15].

$P L T / W B C$ in A-PRF and L-PRF $=P L T / W B C$ in whole blood$[(P L T / W B C$ in red clot)-(PLT/WBC in the serum over the clot of $P R F)-(P L T / W B C$ in the supernatant after compression of the clot PRF)]

The examinations were performed with a Cell Dyn $3500 \mathrm{R}$ cell counter (ABBOTT).

\section{Statistical analysis}

The statistical significance for the differences between two groups was calculated using Student mins $t$-test for repeated measures for parametric variables and with the $\chi^{2}$ for non-parametric variables.

$P$ values $<0.005$ were considered statistically significant.

The data were analysed using the Discipline Biomedical Statistics of Stanton-Glantz 2007 software package, version 6.0 .

\section{Results}

The procedure was well-tolerated in all animals. No significant differences were found in the basic haematological confrontation, which had an average concentration of RBC $9.8 \times 10^{6} / \mathrm{mL}$ (range: $\left.7-13\right)$, WBC $5.1 \times 10^{3} / \mathrm{mL}$ (range: $\left.5-13\right)( \pm$ 0.37 C.I. $95 \%)(p=0.24)$ and a platelet count average of 106.8 $\times 10^{3} / \mathrm{mL}$ (range: $\left.100-350\right)$ ( \pm 15.3 I.C. $95 \%$ ) $(p=0.5$ ). It was not possible to directly quantify the platelet concentration and WBC trapped inside the L-PRF clot, which was derived indirectly by comparing the mean values of whole blood, the mean values of the supernatant obtained after compression of the clot at $2 \mathrm{~min}$, and the average values obtained with the counts of smears of the red clot after the removal of the L-PRF clot.

Table 1 shows the number of leukocytes, red blood cells and platelets in the whole blood (control group) and red blood clot after collection of the PRF membrane (test group) responsible for therapeutic potentiation.

Table 2 compares the characteristics of clots and membranes of PRF obtained in humans (centrifugal Intraspin) reported by Pinto, et al. [16] and those we observed in the horse model (centrifuge DUO). In this comparison, it was verified that there are significant differences in the characteristics of the clot (weight), but these differences are eliminated when the membranes derived from the compression were examined. This observation, in our opinion, would be assigned to a different content of exudate (weight of the exudate is $1.47 \pm 0.13 \mathrm{~g}$ in humans, $3.05 \pm 0.11 \mathrm{~g}$ in horses, $p=0.000$ ).

In this study, the size of the membranes is not found to be in relation to the haemoglobin content or the content of erythrocytes encountered in the blood count baseline. In optical microscopy (Figure 3), most of the cell bodies (stained in dark purple for the nuclei) were concentrated in the proximal part (head) of each membrane, the last $1 / 4$ was observed at the centre, and the distal part had only residual traces of cell bodies. Optical microscopy has not, however, allowed the observation of the exact state of these cell bodies in greater detail.

The results of the blood counts of whole blood and of the supernatant obtained from the clot after 0 min of compres-

Table 1: Leukocytes, RBC and Platelets number in whole blood (control group) and red clot after PRF membrane collecting (test group).

\begin{tabular}{|c|c|c|c|c|c|c|}
\hline & \multicolumn{2}{|c|}{ Leukocytes/ $\mu$ l } & \multicolumn{2}{|l|}{$\mathrm{RBC} / \mu \mathrm{l}$} & \multicolumn{2}{|c|}{ Platelets/ $\mu \mathrm{l}$} \\
\hline & Mean & Range & Mean & Range & Mean & Range \\
\hline Control & 6.900 & $6.100-7.800$ & $5.19\left(10^{6}\right)$ & $5.01-5.52\left(10^{6}\right)$ & $2.66\left(10^{5}\right)$ & $2.18-3.09\left(10^{5}\right)$ \\
\hline Group 1 & 3.500 & $3.000-3.800$ & $5.89\left(10^{6}\right)$ & $5.75-6.08\left(10^{6}\right)$ & 6.000 & $4.000-8000$ \\
\hline Group 2 & 3.600 & $3.300-4.000$ & $5.84\left(10^{6}\right)$ & $5.78-5.91\left(10^{6}\right)$ & 7.000 & $6.000-9000$ \\
\hline
\end{tabular}


Citation: Crisci A (2019) The L-PRF Membrane (Fibrin Rich in Platelets and Leukocytes) and Its Derivatives (A-PRF, I-PRF) Are Useful as a Source of Stem Cells in Regenerative Wound Therapy: Experimental Work on the Horse. Regen Med Ther 3(1):37-45

Table 2: Comparison of membranes obtained from human blood (Pinto, et al. [16]) and from equine blood.

\begin{tabular}{|c|c|c|c|c|}
\hline \multirow[b]{2}{*}{ Variable } & $\operatorname{Man}(n=8)$ & Horse $(n=6)$ & \multirow[b]{2}{*}{ Student mins $t$} & \multirow[b]{2}{*}{ Significance } \\
\hline & \multicolumn{2}{|l|}{ Average ( \pm SD) } & & \\
\hline Final $\mathrm{T}^{\circ}$ test tube $\left({ }^{\circ} \mathrm{C}\right)$ & $27.5( \pm 0.66)$ & & & \\
\hline Weight of the clot $(\mathrm{g})^{*}$ & $2.09( \pm 0.19)$ & $4.23( \pm 0.55)$ & $p=0.000<0.005$ & $\mathrm{~S}$ \\
\hline Weight of the membrane (g) & $0.62( \pm 0.15)$ & $0.78( \pm 0.08)$ & $p=0.036>0.005$ & NS \\
\hline Exudate weight (g) & $1.47( \pm 0.13)$ & $3.05( \pm 0.11)$ & $p=0.000<0.005$ & $S$ \\
\hline Length of the clot (mm) & $35.69( \pm 3.43)$ & $44.38( \pm 3.83)$ & $p=0.000<0.005$ & S \\
\hline Width of the clot (mm) & $12.81( \pm 0.75)$ & $14.74( \pm 1.23)$ & $p=0.003<0.005$ & S \\
\hline Height of the clot (mm) & & $7.02( \pm 1.09)$ & & \\
\hline Surface area of the clot $\left(\mathrm{mm}^{2}\right)$ & & $4.10( \pm 0.86)$ & & \\
\hline Length of the membrane ( $\mathrm{mm}$ ) & $34.81( \pm 2.95)$ & $36.81( \pm 3.18) \mathbb{4}$ & $p=0.248>0.005$ & NS \\
\hline Width of the membrane $(\mathrm{mm})$ & $12.25( \pm 0.71)$ & $13.02( \pm 1.01) \mathbb{4}$ & $p=0.119>0.005$ & NS \\
\hline Height of the membrane $(\mathrm{mm})$ & & $3.02( \pm 0.51) \mathbb{\mathbb { Q }}$ & & \\
\hline Surface area of the membrane $\left(\mathrm{cm}^{2}\right)$ & & $3.08( \pm 0.5) \mathbb{1}$ & & \\
\hline Weight ratio clot/blood sample (\%) $10 \mathrm{ml}$ & $20.94( \pm 2.4)$ & $32.53( \pm 0.54)$ & $P=0.000<0.005$ & S \\
\hline
\end{tabular}

${ }^{*}$ The difference of the weight clot is due to a difference of exudate content; $\mathbb{1}$ Average values $\left( \pm\right.$ D.S.) after 2 min compression at $30^{\circ} \mathrm{C}$; N.B.: The values are not in relation to the content of $\mathrm{Hb}$ and erythrocytes in whole blood.

Table 3: Results of the blood counts of whole blood, of the supernatant obtained by compression of the clot at 0 min compared with the counts of erythrocytes, platelets, and WBC of the red clot smear at $0 \mathrm{~min}$, with tests of significance.

\begin{tabular}{|c|c|c|c|c|c|c|c|c|}
\hline \multirow{3}{*}{\begin{tabular}{|l|} 
Type \\
RBC \\
\end{tabular}} & \multirow{2}{*}{\multicolumn{2}{|c|}{$\begin{array}{l}\text { CBC blood } \\
\text { Average } \pm \text { D.S. }\end{array}$}} & \multirow{2}{*}{$\begin{array}{l}\text { CBC supernatant at } \\
0 \text { min } \\
\text { Average } \pm \text { D.S. }\end{array}$} & \multirow{3}{*}{$\begin{array}{l}\text { Smear C.R. } 0 \text { min } \\
\text { Average } \pm \text { D.S. } \\
7.399 .440 \pm 27.039 .76 \\
\end{array}$} & \multicolumn{4}{|c|}{ Between CBC blood and supernatant 0 min } \\
\hline & & & & & \multicolumn{2}{|l|}{$t$-test ${ }^{*}$} & \multicolumn{2}{|l|}{$x^{2}$} \\
\hline & \multicolumn{2}{|c|}{$7.648 .000 \pm 11.309 .81$} & $13.428 \pm 21345$ & & $p=0.411>0.05$ & NS & $p=0.000<0.005$ & S \\
\hline WBC & \multicolumn{2}{|l|}{$5150 \pm 369$} & $30 \pm 27.99$ & $8.5 \pm 2.12$ & $p=0.255>0.05$ & NS & $p=0.000<0.005$ & S \\
\hline PLT & \multicolumn{2}{|c|}{$106.780 \pm 153.51$} & $479 \pm 77.614$ & $500 \pm 707.11$ & $p=0.031<0.05$ & S & $p=0.000<0.005$ & S \\
\hline Neutrophil & \multicolumn{2}{|l|}{$3046 \pm 857$} & $0.29 \pm 0.76$ & & $p=0.280>0.05$ & NS & $p=0.991>0.005$ & NS \\
\hline Basophils & \multicolumn{2}{|l|}{$4.2 \pm 1.3$} & $2.29 \pm 2.14$ & & $p=0.785>0.05$ & NS & $p=0.611>0.005$ & NS \\
\hline Lynphocites & \multicolumn{2}{|l|}{$1606 \pm 668$} & $19 \pm 23.15$ & & $p=0.238>0.05$ & NS & $p=0.000<0.005$ & $S$ \\
\hline Monocytes & \multicolumn{2}{|l|}{$490.2 \pm 138.06$} & $4.57 \pm 7.68$ & & $p=0.631>0.05$ & NS & $p=0.928>0.005$ & NS \\
\hline Eosinophils & \multicolumn{2}{|l|}{$5.4 \pm 5.37$} & $4 \pm 9.71$ & & $p=0.906>0.05$ & NS & $p=0.316>0.005$ & NS \\
\hline \multirow[t]{2}{*}{ Type } & \multicolumn{8}{|c|}{$\begin{array}{l}\text { Membrane L-PRF/A-PRF } \\
0 \mathrm{~min}\end{array}$} \\
\hline & No./$/ \mu l$ & \multicolumn{3}{|l|}{$\%$} & & & & \\
\hline RBC & 21.6012 & \multicolumn{2}{|l|}{$0.00 \%$} & & & & & \\
\hline WBC & 5111.15 & \multicolumn{2}{|c|}{$99.24 \%$} & & & & & \\
\hline PLT & 105.801 & \multicolumn{2}{|c|}{$99.00 \%$} & & & & & \\
\hline
\end{tabular}

*processing performed on two comparisons. Hypothetical content of RBC, WBC, PLT in the L-PRF membranes at 0 min and 60 min with significance tests. $P>0.05=+0.5 \%$ non-significant difference; $p<0.01=-1 \%$ significant difference.

sion are compared with the counts of erythrocytes, platelets, and the WBC smear of the red clot after 0 min, and are shown with the corresponding statistical tests in Table 3.

Table 3 demonstrates the statistically significant difference between the content of RBC, WBC, and platelets between the supernatant derived from the compression of the clot at $0^{\prime}$ and the values obtained with the blood count. There is also shows a statistically significant difference between the content of RBC, WBC, and PLT in smears obtained from the red clot at $0 \mathrm{~min}$, as shown in Figures $3 \mathrm{~A}-\mathrm{C}$ at various magnifications. Table 3 also shows the hypothetical contents of RBC, WBC, and PLT in L-PRF/A-PRF membranes derived from the difference of these corpuscular elements between whole blood, the supernatant at $0 \mathrm{~min}$, and the smear of the red clot at $0 \mathrm{~min}$. The $t$-test of Student shows significant differences between RBCs at 0 min and PLT at 0 min in L-PRF/A-PRF membranes and the blood count test. 
Citation: Crisci A (2019) The L-PRF Membrane (Fibrin Rich in Platelets and Leukocytes) and Its Derivatives (A-PRF, I-PRF) Are Useful as a Source of Stem Cells in Regenerative Wound Therapy: Experimental Work on the Horse. Regen Med Ther 3(1):37-45

The content of RBC in the membranes is $0.0028 \%$, that of WBC is $99.24 \%$, and that of PLT is $99.0 \%$, compared to the content in whole blood.

\section{Discussion}

The study performed by McLellan, et al. [17] has shown that the equine PRF is similar to that of humans, providing an immediate and constant source of tissue growth factors. Our study has attempted to standardize the preparation of the L-PRF/A-PRF procedure which, while remaining a technique of easy execution and low-cost, does not require specialized equipment, but has a certain constancy in the production of a membrane in terms of L-PRF/A-PRF macroscopic and microscopic features. The autologous platelet concentrates are promising in the field of regenerative medicine due to the abundance of growth factors.

The L-PRF represents a very significant advance in the evolution of platelet concentrates since it is essentially a fibrin membrane with platelets and leukocytes trapped within along with stem cells. These solid membranes possess excellent handling characteristics, and can be firmly sutured in an anatomically-desired location during open surgeries. However, the physical and biological properties are relatively unknown and have yet to be fully studied.

The L-PRF/A-PRF will form when the steps described above are strictly observed.

One of the important considerations in generating a good membrane is the delay in the time between blood collection and centrifugation, as well as the processing temperature. The success of the technique depends entirely on the speed of collection of blood and the immediate transfer into a centrifuge, usually within one minute, and by a centrifugation temperature and higher squeeze at $21{ }^{\circ} \mathrm{C}$ (between 21 and $\left.30^{\circ} \mathrm{C}\right)$.

You cannot generate a clot of well-structured L-PRF/APRF (with its specific cellular content, architecture of the matrix, and profile of the release of growth factors) if the collection of blood is prolonged and not homogeneous, or if the centrifugation temperature is below $21^{\circ} \mathrm{C}$ or more than $30{ }^{\circ} \mathrm{C}$; instead, it will form an inconsistent, crumbly mass of fibrin with unknown contents.

The L-PRF/A-PRF functions as a provisional extracellular matrix, which is transformed into functional tissue during healing, and can be subjected to mechanical forces and healing outcomes with success, which depends on the structural integrity and, therefore, it is important to clarify its physical properties. The L-PRF/A-PRFs look like dense connective tissue with superior handling characteristics. Therefore it is assumed that, as in the L-PRF, also in the A-PRF there is a low rigidity.

With an elastic modulus of $0.470 \mathrm{MPa}(\mathrm{SD} \pm 0.107)$ the L-PRF membrane stretches to twice its initial length before breakage (of $215 \%$ strain). These data confirm the published literature [18] who reported a low rigidity (1-10 MPa) and a high voltage (up to $150 \%$ ) before breaking down.

On the basis of these results, it is clear that $L-P R F / A-P R F$ is a new biomaterial with unique features: The anticipated prepa- ration of autologous blood, the simplicity of the protocol, the defined architecture, the impressive mechanical properties, and the abundance of derived growth factors from activated platelets. Our experiments on equine blood will no doubt be able to improve our understanding of healing, as well as contribute to advancing the field of personalized medicine.

Limitations that were found in the clinical setting and usage of these products include:

1) Since PRF is an autologous product, an increased requirement for the biomaterial availability is difficultly achieved. Therefore, its use in surgical procedures must be tightly controlled.

2) PRF contains circulating immune cells, as well as antigenic molecules that prevent its usage as allogenic material; an increased risk for the transmission of infectious diseases is also to be taken into account.

At this point, among the different parameters that were not included in this type of classification, we recognize: Platelet concentration, leukocyte concentration and the proportional amount of the different leukocyte types. Platelet concentration-related problems are non-existent, as all platelets included in the blood sample are activated and integrated inside the clot's fibrin matrix.

Concerning the leukocytes' count and concentration, their influence should be studied with particular care, as their presence or absence could explain the conflicting results we observed.

In a recent study by Kitamura $Y$, et al. [19] shows a method for direct estimation of platelet count in PRF. These authors used a commercially available recombinant t-PA, Alteplase (GRTPA ${ }^{\circledR}$; Mitsubishi Tanabe Pharma Corp., Osaka, Japan) through a digestion method. Here, they have shown that t-PA is powerful enough to be able to count dispersed platelets aggregated into platelet-enriched insoluble fibrin matrices.

\section{Conclusion}

Concluding this research, we can affirm that, to achieve a standard procedure for PRF preparation as graft material for tissue regeneration purposes, we suggest the employment of PRF membrane's region with the highest possible platelet enrichment and, moreover, we suggest to avoid squeezing all of the PRF clot plasma. Hence, it is advisable to compress the clot with a compression device (L-PRF Wound Box). It's difficult, therefore, to control precisely the human-derived materials' quality, like PRF preparations, but is it important to apply the highest possible quality-control check on PRF preparations before their clinical application.

Currently, their delivery relies on poorly controlled bulk release. As consequence, prolonged treatments require multiple treatments e.g. numerous injections $i$-PRF. This results in strongly fluctuating growth factor concentrations, which impairs clinical predictability. Biomaterials can act as controlled release devices, which will allow for sustained or even on-demand delivery of these growth factor cocktails. In addition, it can be envisioned that biomaterials can covalently bind 
Citation: Crisci A (2019) The L-PRF Membrane (Fibrin Rich in Platelets and Leukocytes) and Its Derivatives (A-PRF, I-PRF) Are Useful as a Source of Stem Cells in Regenerative Wound Therapy: Experimental Work on the Horse. Regen Med Ther 3(1):37-45

specific growth factors to locally retain high levels of these molecules.

Further clinical, histological and statistical studies are required to understand the benefits of this new platelet concentration technique. However, we cannot sweep aside the fact that, when obtained from an autologous blood sample, produced PRF is scarce and only a limited volume can be used. This is a limitation for systematic PRF usage in Regenerative Surgery interventions. Even if the potential applications of PRF are ample, an accurate knowledge of the biomaterial is necessary, including information on its biology, efficacy and limits, to optimize its usage in everyday clinical practice.

Cell migration plays a crucial role in the healing process. MSCs represent a cell pool, able to reconstruct the damaged tissue, and endothelial cells contribute to angiogenesis. Migration models induced by the supernatant of platelet concentrate's culture do not differ between the two types of cells. The stronger MSCs and HUVECs migration was observed as a reply to $L$-PRF. All of the above signifies that $L$-PRF could be useful as a healing biomaterial, and as a natural anti-hemorrhagic agent to be used at surgical sites [20].

\section{References}

1. Pretorius E, Briedenhann S, Marx J, et al. (2007) Ultrastructural comparison of the morphology of three different platelet and fibrin fiber preparations. Anat Rec 290: 188-198.

2. J Choukroun, F Adda, C Schoeffler, et al. (2001) Une opportunite en paro-implantologie: le PRF. Implantodontie 55-62.

3. Crisci A, De Crescenzo U, Crisci M (2018) Platelet-rich concentrates (L-PRF, PRP) in tissue regeneration: Control of apoptosis and interactions with regenerative cells. J Clin Mol Med 1: 5-12.

4. Litvinov RI, Weisel JW (2016) What Is the biological and clinical relevance of fibrin? Semin Thromb Hemost 42: 333-343.

5. Soloviev DA, Hazen SL, Szpak D, et al. (2014) Dual role of the leukocyte integrin $\alpha \mathrm{M} \beta 2$ in angiogenesis. J Immunol 193: 47124721.

6. Crisci A, Lombardi D, Serra E, et al. (2017) Standardized protocol proposed for clinical use of L-PRF and the use of L-PRF Wound Box $^{\circledR}$. J Unexplored Med Data 2: 77-87.

7. Ghanaati S, Booms P, Orlowska A, et al. (2014) Advanced plateletrich fibrin: $A$ new concept for cell- based tissue engineering by means of inflammatory cells. J Oral Implantol 40: 679-689.

8. Choukroun J (2014) Advanced-PRF and i-PRF: Platelet concentrates or blood concentrates? J Periodont Med Clin Pract 1: 1-3.
9. Miron RJ, Fujioka-Kobayashi M, Hernandez M, et al. (2017) Injectable platelet rich fibrin (i-PRF): Opportunities in regenerative dentistry? Clin Oral Invest 21: 2619-2627.

10. Kobayashi M, Kawase T, Horimizu M, et al. (2012) A proposed protocol for the standardized preparation of PRF membranes for clinical use. Biologicals 40: 323-329.

11. Dohan Ehrenfest DM, Bielecki T, Jimbo R, et al. (2012) Do the fibrin architecture and leukocyte content influence the growth factor release of platelet concentrates? An evidence-based answer comparing a pure platelet-rich plasma (P-PRP) gel and a leukocyte- and platelet-rich fibrin (L-PRF). Curr Pharm Biotechnol 13: $1145-1152$.

12. Crisci A, Benincasa G, Crisci M, et al. (2018) Leukocyte plateletrich fibrin (L-PRF), a new biomembrane useful in tissue repair: Basic science and literature review. Biointerface Res Appl Chem 8: 3635-3643.

13. Crisci A, Marotta G, Licito A, et al. (2018) Use of leukocyte platelet (L-PRF) rich fibrin in diabetic foot ulcer with osteomyelitis (Three clinical cases report). Diseases 6: 30.

14. Crisci A, Crisci M, Boccalone E (2014) Final results of an experimental research about a technique of measurement of skin lesions. Esperienze Dermatologiche 16: 147-152.

15. Watanabe T, Isobe K, Suzuki T, et al. (2017) An evaluation of the accuracy of the subtraction method used for determining platelet counts in advanced platelet-rich fibrin and concentrated growth factor preparations, Dent J 5: 7.

16. Nelson R Pinto, Andrea Pereda, Paula Jiménez, et al. (2014) The impact of the centrifuge characteristics and centrifugation protocols on the cells, growth factors and fibrin architecture of a leukocyte-and platelet-rich fibrin (L-PRF) clot and membrane. Part 2: macroscopic, photonic microscopy and Scanning Electron Microscopy analysis of 4 kinds of L-PRF clots and memebranes. POSEIDO 2: 141-154.

17. McLellan J, Plevin S (2014) Temporal release of growth factors from platelet-rich fibrin (PRF) and platelet-rich Plasma (PRP) in the horse: A comparative in vitro analysis. Intern J Appl Res Vet Med 1: 48-57.

18. Madurantakam P, Yoganarasimha S, Hasan FK (2015) Characterization of leukocyte-platelet rich Fibrin, a novel biomaterial. J Vis Exp.

19. Kitamura $Y$, Watanabe $T$, Nakamura $M$, et al. (2018) Platelet counts in insoluble platelet-rich fibrin clots: A direct method for accurate determination. Front Bioeng Biotechnol 6: 4 .

20. Crisci A, Manfredi S, Crisci M (2019) Fibrin rich in leukocyteplatelets (L-PRF) and injectable fibrin rich platelets (i-prf), two opportunity in regenerative surgery: Review of the sciences and literature. IOSR-JDMS 18: 66-79.

DOI: $10.36959 / 654 / 392$

Copyright: @ 2019 Crisci A. This is an open-access article distributed under the terms of the Creative Commons Attribution License, which permits unrestricted use, distribution, and reproduction in any medium, provided the original author and source are credited. 